\title{
FATORES INDUTORES À INOVAÇÃO TECNOLÓGICA DA INDÚSTRIA MADEIREIRA DA REGIÃO DE LAGES/SC
}

\author{
Flávio José Simioni \\ Doutor em Engenharia Florestal pela Universidade Federal do Paraná - UFPR \\ Professor da Universidade do Estado de Santa Catarina - UDESC \\ flavio.simioni@udesc.br (Brasil) \\ Debora Nayar Hoff \\ Doutora em Agronegócios pela Universidade Federal do Rio Grande do Sul - UFRGS \\ Professor da Universidade Federal do Pampa - UNIPAMPA \\ deborahoff@ie.ufu.br (Brasil)

\section{Erlaine Binotto} \\ Doutora em Agronegócios pela Universidade Federal do Rio Grande do Sul - UFRGS \\ Professor da Universidade Federal da Grande Dourados - UFGD \\ e-binotto@uol.com.br (Brasil)
}

\section{RESUMO}

Objetivou-se analisar as variáveis que interferem na adoção de inovações tecnológicas de empresas madeireiras da região de Lages/SC, suas características e a conduta padrão das firmas quanto à inovação. A pesquisa contemplou 36 empresas e os dados foram coletados via questionário e submetidos à análise de regressão logística (logit) e à Principal Component Analysis (PCA). Os resultados indicam que a inovação está associada à localização em um ambiente externo mais competitivo, ao aumento do tempo de vida, à utilização de crédito e à busca da certificação pelas empresas, enquanto que as serrarias estão associadas à não inovação. As tecnologias adotadas são amplamente difundidas no setor.

Palavras-chave: Tecnologia; Indústria; Madeira; Path dependence. 


\section{INTRODUÇÃO}

A inovação tecnológica na indústria madeireira tem sido foco de debates no meio empresarial, visando maior eficiência na utilização da matéria prima, atendimento das questões ambientais e competitividade setorial. Na região de Lages/SC, estas questões ganham relevância, dado que a indústria madeireira está inserida em um complexo produtivo baseado em florestas plantadas de pinus, cuja finalidade principal é a produção de celulose e papel, com excedente alimentando outras cadeias do complexo produtivo (SIMIONI et al., 2007).

Hoff e Simioni (2004) destacaram que, regionalmente, a baixa utilização de tecnologias avançadas é um dos limitantes ao desenvolvimento da indústria. Uma das características que reforça essa limitação é a manutenção do perfil produtivo, ou seja, comparando-se os dados de 2009 (SIMIONI et al., 2010) em relação a 2002 (SIMIONI et al., 2002), mais de 50\% da produção regional continua sendo a madeira serrada. Considerando que este segmento produtivo tende à baixa utilização de tecnologias, à presença de alta geração de resíduos e à grande variabilidade dos padrões do produto final, a expectativa é que comprometa a competitividade das empresas que dele dependem. Por outro lado, empresas que precisam qualificar ou diferenciar seu produto final, tendem ao maior uso de tecnologias e adoção de inovações.

Esta percepção torna-se mais relevante quando se considera a realidade competitiva atual, sobretudo a do mercado externo, que requer um perfil de empresa tecnologicamente mais avançada e com maior agregação de valor nos processos e produtos. Condição essa que exige o alcance de patamares mais elevados de competitividade baseados na capacidade de gerar inovações e gerir eficientemente as tecnologias. A questão estabelecida remete para a necessidade de se compreender como se dá o processo de inovação e de gestão da tecnologia neste setor, visando identificar sua presença e características.

Assim, o objetivo deste trabalho foi identificar as variáveis que aumentam a probabilidade das empresas madeireiras adotarem inovações tecnológicas, bem como analisar quais fatores são importantes para sua indução e quais limitam a adoção da inovação tecnológica. Complementarmente, identificou-se o tipo, maturidade, forma de adoção e os efeitos esperados pelos gestores quando da adoção da inovação. Por fim, buscou-se verificar se as empresas acompanham a conduta padrão das firmas do setor e se a realidade encontrada dá indicativos de uma condição path dependence.

Revista de Administração e Inovação, São Paulo, v.12, n.1, p.248-270, jan./mar. 2015. 


\section{INOVAÇÃO TECNOLÓGICA: CONCEITOS E CLASSIFICAÇÕES}

Para contribuir com as análises pretendidas, esta seção apresenta, brevemente, tratamento teórico e analítico sobre tecnologia e inovações tecnológicas e suas relações com a competitividade das empresas.

Neste trabalho, a inovação foi interpretada como: "implementação de um produto (bem ou serviço) novo ou significativamente melhorado, ou um processo, ou um novo método de marketing, ou um novo método organizacional nas práticas de negócios, na organização do local de trabalho ou nas relações externas" (OCDE, 2004, p. 55). Observando-se estudos sobre a inovação na indústria madeireira, em especial Wagner e Hansen (2005), pode-se afirmar que estes usam o mesmo entendimento de inovação.

Quando se discutem os processos de expansão das inovações tecnológicas, existem duas abordagens diferentes sobre os elementos determinantes destes processos: 1) demand pull, que considera as forças de mercado como fator impulsionador; 2) technology push, onde a mudança técnica ocorre a partir do caminho delineado pela própria tecnologia (DOSI, 1988).

$\mathrm{Na}$ perspectiva da demand pull, o mercado produz estímulos para o desenvolvimento de processos inovativos, que resultam em direções da tecnologia. Estes sinais seriam captados a partir das preferências dos consumidores sobre aspectos técnicos dos bens que desejam, ou pelo incremento no desejo por bens com maior tecnologia incorporada, o que está correlacionado, geralmente, com crescimento da renda. A mudança técnica de processos e de produtos é a forma pela qual a firma reage às mudanças, visando atender ao mercado consumidor.

A technology push considera que a tecnologia vigente ajuda a determinar o caminho que as inovações devem seguir, sendo importantes as interações entre a ciência e a tecnologia. Os avanços na ciência pura constituem estimuladores do progresso técnico, cujos resultados geralmente ocorrem de forma autônoma e independente das vontades da sociedade. Com isso, a tecnologia que será utilizada pode ser produzida, distribuída e trocada, sem que tenha sido originada de uma demanda específica, ou seja, a existência de disponibilidade tecnológica pode criar a demanda pela tecnologia (DOSI, 1988; CÁRIO, 1995). Neste sentido, com base em Schumpeter "é a inovação tecnológica que dispara o mecanismo que provoca mudanças no comportamento dos agentes econômicos, realoca recursos, destrói métodos de produção tradicionais e muda, qualitativamente, a estrutura econômica" (SILVA, 1995, p. 44).

Cassiolato (1994) completa, apontando que o processo de mudança tecnológica é uma atividade caracterizada por um contexto seletivo que ocorre através de trajetórias, que são constantemente

Revista de Administração e Inovação, São Paulo, v.12, n.1, p.248-270, jan./mar. 2015. 
interrompidas por descontinuidades associadas com o surgimento de novos paradigmas tecnológicos. Independente do fator impulsionador da mudança tecnológica (mercado ou disponibilidade da tecnologia) existe um percurso quase que padrão para a difusão da inovação.

No âmbito da difusão tecnológica, Nelson e Winter (1982) apontam que a preocupação central da teoria evolucionária é com o processo dinâmico, através do qual a conduta padrão das firmas e os resultados de mercado são conjuntamente determinados ao longo do tempo. Dentro desta concepção, dependendo das condições encontradas no sistema econômico, pode-se ter maior ou menor facilidade para que a inovação tecnológica ocorra. As novas tecnologias centrais nem sempre encontram o marco sócio institucional adequado, assim, sua difusão pode demorar devido às limitações sociais do passado. As mudanças no capital social, o perfil de qualificação, a estrutura industrial e a organização social que exigem a introdução e a difusão das novas tecnologias revolucionárias dependem de tempo (CASSIOLATO, 1994).

Mais que isso, pode-se dizer que existe certo tipo de dependência da inovação na estrutura já instalada no sistema econômico. Dosi e Nelson (1994) chamam atenção para o processo de path dependence, que está inter-relacionado com os processos de mudança e de organização das estruturas industriais, justamente porque estas dependem do caminho que foi trilhado ao longo do tempo e das estruturas já criadas, sejam estas físicas, culturais, sociais, ou de recursos materiais, técnicos ou humanos. Pode-se imaginar que uma tecnologia seja tão mais difícil de ser estabelecida, quanto mais radical é a mudança que ela exige nestas estruturas já existentes.

Segundo Nelson e Winter (1982), a ideia chave do processo da teoria evolucionária é que a condição da indústria em cada período de tempo lança as sementes de sua condição no tempo seguinte, no mesmo sentido da path dependence apontada por Dosi e Nelson (1982). De acordo com os autores, o que a condição industrial de um particular período realmente determina é a distribuição probabilística de sua condição no período seguinte.

De acordo com a visão Schumpeteriana, a economia é baseada num processo ininterrupto de introdução e difusão de inovações em sentido amplo, isto é, de quaisquer mudanças no espaço econômico no qual operam as empresas. Para Schumpeter, a inovação é o resultado da busca constante de lucros extraordinários, mediante obtenção de vantagens competitivas entre os agentes, que procuram diferenciarem-se uns dos outros nas mais variadas dimensões do processo competitivo. Neste sentido, a busca permanente de diferenciação, por meio de estratégias deliberadas, visa à obtenção de vantagens competitivas que proporcionem lucros de monopólio, ainda que temporários (POSSAS, 2002).

Revista de Administração e Inovação, São Paulo, v.12, n.1, p.248-270, jan./mar. 2015. 
A introdução, pelos neo-schumpeterianos, Nelson e Winter (1982), dos termos busca (search) de inovações e de seleção (selection) dos resultados econômicos dessas mesmas inovações, baseia-se na interação temporal entre as estratégias empresariais, que envolvem o referido processo de busca de inovações e de seleção pelo mercado destas. Sendo relevante como objeto de análise a trajetória resultante.

Possas (1993) descreve conceitos elaborados por Dosi (1988) que permitem identificar um ambiente em evolução que caracteriza a construção de vantagens competitivas. De acordo com esta perspectiva teórica, o progresso técnico se dá principalmente com base na busca de respostas às questões colocadas pela prática corrente, no interior de um sistema particular de abordagem dos problemas técnicos.

Existem diversas trajetórias naturais ou tecnológicas, ligadas à noção de aprendizado. As inovações não costumam ocorrer num só golpe, elas são construídas, por exemplo, via learning by doing ou learning by using. Quando se segue uma trajetória natural, as inovações são bastante previsíveis, sendo possível identificar, ao longo do tempo, que linhas gerais deverão ser seguidas. À medida que elas rompem o paradigma, tem-se um maior grau de imprevisibilidade. Diferenciam-se assim as inovações tecnológicas que seguem um caminho previsível das que significam rupturas mais profundas (não esperadas), cercadas de maior grau de incerteza (POSSAS, 1993).

Para entender a manutenção das vantagens competitivas decorrentes da inovação ao longo do tempo, Possas (1993) destaca:

a) Cumulatividade: o progresso técnico segue uma trajetória tecnológica, decorrente do acúmulo de conhecimento, ou seja, a experiência com a utilização da tecnologia leva ao aprendizado e fornece condições para aperfeiçoá-la e seguir inovando. Nesta perspectiva, as firmas que se adiantam são altamente premiadas e ampliam suas vantagens em relação aos que se atrasam. De outra forma, as vantagens competitivas conquistadas geram mais poder para ampliá-las;

b) Apropriabilidade: num ambiente de incerteza, a apropriação de ganhos extraordinários quando ocorre a introdução de avanços é fundamental para estimular a inovação. O grau de incerteza é influenciado pelo aprendizado, que por sua vez leva a uma maior incerteza, pois quanto mais os agentes esperam aprender, mais provável que a inovação seja logo suplantada. A apropriabilidade é ampliada nos casos em que ocorre learning by doing, resultando na curva de aprendizado e, reduzida quando a inovação for mais facilmente imitável ou superável, ou seja, facilmente transmissível. Em síntese, o que determina a 
apropriabilidade de uma inovação é a evolução do grau de diferenciação do produto que ela proporciona, seja pela manutenção de custos inferiores ou de preços mais elevados;

c) Oportunidade: diz respeito às possibilidades de incorporar avanços a um ritmo intenso, gerando um fluxo de novos produtos e processos produtivos, rapidamente substituídos. Para produtos novos existem inúmeras oportunidades de melhorar o processo produtivo, utilizar as formas de aprendizado e explorar as vantagens competitivas. Para produtos difundidos diminuem essas oportunidades, ou seja, as oportunidades inovativas são pequenas e o mercado se estabiliza, tornando-se maduro;

d) Interatividade e Flexibilidade: as vantagens competitivas conquistadas pelo produtor devem ser mantidas. Um dos elementos importantes para isso é a interatividade com fornecedores, por exemplo. Já a flexibilidade possibilita a redução de perdas em situações em que a realidade não confirme as expectativas, ou seja, os resultados esperados para algumas decisões tomadas nem sempre se concretizam. Neste ambiente de incerteza, a flexibilidade se torna importante.

No que tange aos tipos de inovações tecnológicas, de acordo com Tigre (2006), podem ser classificadas de acordo com seu grau de inovação e pela amplitude de suas mudanças. A taxonomia mais utilizada é a dada por Freeman e Soete (1997) onde as mudanças tecnológicas são classificadas em quatro níveis, segundo seus impactos: incremental, radical, novo sistema tecnológico e novo paradigma tecnoeconômico.

As mudanças incrementais são aquelas que ocorrem cotidianamente nas empresas, derivadas principalmente do aprendizado interno e da capacitação acumulada. Melhorias feitas nos processos, em design, no layout e na qualidade são exemplos destas. Já as mudanças consideradas radicais, geralmente são derivadas de atividades de $\mathrm{P} \& \mathrm{D}$ e rompem a trajetória existente dando origem a uma nova rota tecnológica. Difere da anterior por provocar saltos de produtividade. As mudanças que caracterizam um novo sistema tecnológico são mais abrangentes, afetando mais de um setor econômico e dando origem a novas atividades econômicas. De modo mais amplo, mudanças que alteram toda a economia, sejam no aspecto técnico, organizacional como no social, caracterizam uma mudança paradigmática. A descoberta da máquina a vapor e da eletricidade são exemplos de mudança que criaram novos paradigmas tecnoeconômicos.

De acordo com o Manual de Oslo (OCDE, 2004) e Tigre (2006), as inovações podem ser de três tipos: a) De produto: ocorre quando a inovação gera um produto novo ou aprimorado, cujas características principais diferem dos produtos já produzidos pela empresa; b) De processo: é a implantação ou adoção de métodos de produção ou comercialização novos ou significativamente 
aprimorados. Pode envolver mudanças de equipamento, recursos humanos, métodos de trabalho ou uma combinação destes; c) Organizacionais: refere-se a mudanças que ocorrem na estrutura gerencial da empresa. Fazem parte a introdução de estruturas organizacionais, a implantação de técnicas de gerenciamento e de orientações estratégicas. São consideradas como inovação apenas se houver mudanças mensuráveis nos resultados, tais como aumento de produtividade ou vendas.

As firmas usualmente combinam mais de uma fonte de inovação, que podem ter origem interna ou externa à empresa. Segundo Tigre (2006), o desenvolvimento interno de tecnologias pode ser através de atividades de $\mathrm{P} \& \mathrm{D}$, da engenharia reversa ou da experimentação. Por meio das atividades de pesquisa, as empresas podem desenvolver pesquisa básica, com apoio de institutos e universidades visando à obtenção de novos produtos que culminam com um novo sistema tecnológico ou novo paradigma tecnoeconômico. Por outro lado, a pesquisa poderá ter um caráter aplicado ou experimental, visando obter uma solução desejada para problemas mais pontuais. Os projetos de P\&D podem ter origem no mercado (demand pull) ou de uma busca de oportunidade para inovar (technology push).

Outra fonte de inovação são os contratos de transferência de tecnologia, podendo envolver diferentes formas de transferência de conhecimentos, de licenças para fabricação de produtos, aquisição de serviços técnicos e de engenharia. Consiste em uma oportunidade de obter um salto tecnológico nos processos ou produtos (TIGRE, 2006).

Ainda, segundo o autor, a incorporação de tecnologias através de máquinas e equipamentos tem se constituído em importante alternativa de aumento da produtividade. Via esta alternativa, as empresas podem absorver principalmente tecnologias de processo, possibilitando o aumento da escala de produção, melhoria de qualidade, redução de custos, tornando-as mais competitivas.

O conhecimento também é fonte de tecnologia, quando permite não só a invenção, mas o aprimoramento dos processos, produtos e sistemas de gestão, constituindo-se também em vantagem competitiva. As interações entre o conhecimento tácito e o explícito são fundamentais para o bom desempenho dos negócios. Para Polanyi (1967), conhecimento tácito é habilidade das pessoas de conhecerem além daquilo que é expresso pela fala por saberem mais do que podem verbalizar. Enquanto o conhecimento explícito pode ser transmitido através da linguagem, documentos, práticas, treinamentos, estudo formal. Ambos podem se manifestar nos níveis individuais, coletivo e organizacional, pois os seres humanos adquirem conhecimento criando e organizando ativamente as suas próprias experiências.

Weick (1991) e Simon (1957) afirmam que a organização, por si só, não possui capacidade para aprender. A organização aprende por meio da aprendizagem de seus membros e quanto maior for o nível de autoconhecimento, maior será a competência (SWIERINGA; WIERDSMA, 1995). Os

Revista de Administração e Inovação, São Paulo, v.12, n.1, p.248-270, jan./mar. 2015. 
mesmos autores conceituam aprendizagem organizacional como uma mudança do comportamento organizacional, um processo coletivo. Apresentam tal perspectiva como uma competência, "aprender a aprender" e a mudança como uma demonstração do aprendizado. Aprendizagem é uma mudança de conduta. Assim pessoas devem criar e mudar a organização por meio da aprendizagem (SWIERINGA; WIERDSMA, 1995).

$\mathrm{Na}$ organização que aprende, o processo vai além do aspecto individual. Nelson e Winter (1982) e Starbuck (1983) reforçam essa ideia ao considerarem que a evolução das organizações ocorre pela sua capacidade de aprender. As organizações têm sistema cognitivo e memória (HEDBERG, 1981; WALSH; UNGSON, 1991); as rotinas e procedimentos trazem consigo histórias (NELSON; WINTER, 1982). O meio pelo qual o conhecimento é integrado depende da história e da cultura do grupo e da organização (BARNEY, 1986).

Nesta discussão surge o conceito de aprendizagem tecnológica que, para Figueiredo (2004), precisa ser entendida em dois sentidos: a) A trajetória de acumulação de capacidade tecnológica, que pode se alterar no decorrer do tempo, no que se a refere à direção dada e a velocidade; b) Os conhecimentos das pessoas que são transformados em produtos físicos, processos, procedimentos, etc., e muitas vezes são tácitos. A transferência de conhecimentos para práticas concretas permite as organizações aumentarem sua capacidade tecnológica.

Figueiredo (2004, p. 6) conceitua capacidade tecnológica a partir de vários autores. As definições consideradas mais antigas dizem respeito a uma "atividade inventiva" ou ao esforço criativo sistemático para obter novos conhecimentos em nível da produção (KATZ, 1976). A capacidade tecnológica, também inclui as aptidões e os conhecimentos incorporados nos trabalhadores, nas instalações e nos sistemas organizacionais, visando mudanças tanto na produção quanto nas técnicas utilizadas (BELL, 1982; SCOTT-KEMMIS, 1988). Dessa forma, poderá ser incrementada via aprendizado tornando-se uma das fontes de competitividade.

\section{METODOLOGIA}

A pesquisa teve como área de abrangência as empresas do setor madeireiro, situadas na região de Lages/SC, sendo parte de uma investigação mais ampla conduzida pelos autores. Consideraram-se empresas madeireiras aquelas que atuam na transformação primária (desdobro) e secundária (beneficiamento) de madeira sólida.

Revista de Administração e Inovação, São Paulo, v.12, n.1, p.248-270, jan./mar. 2015. 
Uma população de 95 empresas foi identificada nos cadastros de empresas junto ao sindicato das indústrias madeireiras (Sindimadeira), nas prefeituras e nas associações dos municípios. A amostra foi definida seguindo critérios de proporcionalidade, acessibilidade e disponibilidade dos sujeitos pesquisados (proprietários ou gerentes do processo produtivo) responderem o questionário, chegando a 36 empresas pesquisadas. Os municípios que tiveram mais empresas pesquisadas foram Lages com $33 \%$ do total, Curitibanos com 22\% e Campo Belo do Sul com 17\%, todas situadas na Serra Catarinense, região de Lages/SC. O perfil das empresas pesquisadas e uma caracterização geral das inovações tecnológicas podem ser observados em Simioni, Binotto e Hoff (2012).

Os instrumentos para a coleta de dados e informações foram um questionário e entrevista estruturados, envolvendo questões relacionadas à inovação tecnológica. As variáveis de análise estudadas foram: perfil das empresas pesquisadas, entendimento de inovação e tecnologia, inovação em produto, processo e gestão, limitadores e indutores da adoção de novas tecnologias, grau da inovação, extensão das mudanças e maturidade das tecnologias empregadas.

As empresas, inicialmente, foram classificadas em duas categorias (grupos): INOVA e NÃO INOVA. As empresas classificadas como INOVA são aquelas que realizaram alguma inovação, de produto ou de processo, durante os três anos que antecederam a pesquisa (2006, 2007 e 2008), enquanto que as classificadas como NÃO INOVA não efetivaram nenhuma inovação no período de análise. Os dados foram submetidos à construção de um modelo de regressão logística (logit), o qual permite realizar predição para variáveis dependentes dicotômicas a partir de um conjunto de variáveis explicativas, conforme metodologia descrita por Ribas e Vieira (2011). Com essa regressão foi possível identificar quais variáveis, internas ou externas às empresas madeireiras, aumentam a probabilidade dos gestores adotarem inovações tecnológicas. As estimativas de probabilidade situamse dentro do intervalo zero e um e, como a estimação não é linear, dado que o efeito marginal de uma variável sobre a probabilidade depende do comportamento de outras variáveis, o efeito marginal pode ser especificado pela equação abaixo:

$$
p=\operatorname{Pr}[Y=1 \mid X=x]=\frac{e^{\beta^{\prime} x}}{1+e^{\beta^{\prime} x}}=\frac{1}{1+e^{-\beta^{\prime} x}}
$$

Onde, $Y$ é a variável dependente e indica a adoção de inovação tecnológica pela indústria madeireira $(0=$ não; $1=$ sim $), X$ é um conjunto de variáveis independentes que influenciam $Y, P r$ é a probabilidade da adoção de uma inovação tecnológica e, $\beta$ é um conjunto de parâmetros estimados.

As variáveis independentes com suas respectivas hipóteses foram:

Revista de Administração e Inovação, São Paulo, v.12, n.1, p.248-270, jan./mar. 2015. 
a) Escolaridade: a variável foi medida considerando o nível de escolaridade do gestor da empresa, atribuindo-se zero para os casos em que o respondente apresentava ensino fundamental incompleto ou completo, um para o ensino médio incompleto ou completo e dois para o ensino superior incompleto ou completo. Espera-se um efeito marginal positivo desta variável, indicando que um nível escolar elevado facilita a absorção e compreensão de novas tecnologias, aumentando assim a probabilidade de adoção da inovação tecnológica na indústria madeireira.

b) Idade da empresa: foi identificada considerando o tempo decorrente do ano da sua fundação até a data da realização da pesquisa. Espera-se um efeito marginal positivo desta variável, ou seja, as empresas com maior idade possuem uma trajetória de crescimento e desenvolvimento que aumentam a probabilidade de adoção da inovação tecnológica na indústria madeireira.

c) Número de funcionários: foi medido em termos absolutos. Espera-se um efeito marginal positivo desta variável, na medida em que empresas de maior porte possuem maior capital investido e, por esta razão, possuem menor aversão ao risco e maior inserção nos mercados, características que aumentam a probabilidade de adoção da inovação tecnológica.

d) Serraria: indica se a empresa atua com o desdobro primário de madeira. A variável é binária (dummy) e admite valor um para as empresas classificadas como serraria e zero para as demais empresas. Desta espera-se um efeito marginal negativo, uma vez que empresas que atuam neste segmento são, tradicionalmente, caracterizadas pela baixa inovação tecnológica.

e) Tamanho: é ordinal e foi identificada considerando o faturamento da empresa. Foi atribuído valor zero para microempresa (ME), um para empresa de pequeno porte (EPP) e dois para empresa de grande porte. Tal como na variável número de funcionários, esperase um efeito marginal positivo para a adoção de inovação tecnológica.

f) Mercado internacional: indica se a empresa atua comercializando seus produtos no mercado externo. A variável é binária (dummy) e admite valor zero para as empresas que não atuam no mercado internacional e um para as que atuam. Espera-se um efeito marginal positivo desta variável, indicando que as empresas que estão inseridas no mercado externo buscam atender normas internacionais e estão mais alinhadas aos padrões de competitividade, aumentando assim a probabilidade de adoção da inovação tecnológica. 
g) Certificação: é dada pela obtenção de certificação da empresa em alguma entidade certificadora, ou seja, possui o Forest Stewardship Council (FSC), Certificação Florestal (CERFLOR) e sistemas de gestão de qualidade (normas ISO, NBR, dentre outras). A variável é binária (dummy) e admite valor zero para as empresas que não possuem certificação e um para as que são detentoras de pelo menos uma certificação. $O$ efeito marginal esperado é positivo, indicando que as empresas certificadas são mais propensas à adoção de inovações tecnológicas com o objetivo de obter e manter os selos ou certificados.

h) Localização: indica se a empresa está sediada no município de Lages ou fora dele. A variável é binária (dummy) e admite valor zero para as empresas que não estão situadas em Lages e um para as sediadas em Lages. Para as empresas localizadas em Lages, espera-se um efeito marginal positivo, indicando que a maior disponibilidade de mão de obra qualificada, acesso a informações e maiores possibilidade de efetivação de parcerias com instituições públicas e privadas visando o desenvolvimento de produtos e processos aumenta a probabilidade de adoção da inovação tecnológica.

i) Crédito: indica se a empresa utiliza linhas de crédito de fontes externas, além do seu capital próprio, para a realização de investimentos. A variável é binária (dummy) e admite valor zero para as empresas que não acessam crédito e um para as que utilizam crédito. Espera-se um efeito marginal positivo, indicando que as empresas que utilizam crédito externo, aumentam sua capacidade de implementar alguma inovação tecnológica.

Os dados também foram submetidos a métodos multivariados de análise estatística, com o objetivo de identificar quais variáveis independentes contribuem para explicar o comportamento da inovação tecnológica nas empresas, dando igual ênfase a todas ao mesmo tempo. As variáveis independentes foram utilizadas para obtenção do comprimento do gradiente e para a seleção do modelo de resposta conforme metodologia descrita por Ter Braak e Smilauer (1998). Como este gradiente foi menor do que três (resposta linear), optou-se pela PCA (Principal Component Analysis).

Complementarmente ao um conjunto de variáveis, foi coletado um conjunto de informações das empresas que adotaram inovações tecnológicas, tais como o tipo e maturidade das inovações, fatores indutores e limitadores, os efeitos esperados, fontes de inovação e formas de aprendizado, dentre outras, a partir das quais se procurou entender o padrão de comportamento do setor.

Revista de Administração e Inovação, São Paulo, v.12, n.1, p.248-270, jan./mar. 2015. 


\section{RESULTADOS E DISCUSSão}

Iniciando pela caracterização geral das empresas, das 36 empresas pesquisadas onze relataram alguma inovação no processo produtivo, representando 30,6\% da amostra. A principal forma de inovação ocorreu por meio da introdução de máquinas e equipamentos mais modernos e automatizados no processo produtivo, visando aumentar a produtividade, a qualidade e a eficiência, a partir do melhor aproveitamento da matéria prima. De acordo com os dados da Pesquisa Industrial de Inovação Tecnológica (PINTEC), realizada pelo IBGE, considerando o período 2006-2008, a taxa de inovação geral da indústria brasileira foi de $38,6 \%$ e de $23,6 \%$ para o segmento de fabricação de produtos de madeira (IBGE, 2010). Isso faz com que a amostra replique localmente uma constatação de âmbito mais amplo para a indústria nacional, estando um pouco acima do indicador específico do setor observado pela PINTEC.

A inovação em produto foi verificada em cinco empresas quando considerado o lançamento de novos produtos nos últimos três anos, reduzindo-se para três empresas se considerados os últimos dois anos e para duas no último ano. Na maioria dos casos, o conhecimento destas possibilidades de inovação é obtido em feiras especializadas do setor florestal e madeireiro (learning by imiting). Observando-se a lista de novos produtos apresentados pelas empresas (lâminas e modelos de portas, embalagens para vinhos, decks, peças e artefatos de madeira), constata-se que estes não representam novidade para o mercado, ficando a inovação restrita no âmbito das empresas.

A análise preliminar dos dados (Tabela 1) revela que as empresas que inovaram (onze) possuem gestores com nível de escolaridade, idade e número de funcionários maiores, não atuam no ramo de serraria e estão localizadas no município de Lages. Enquanto que as que não inovaram, estão localizadas no interior, atuando principalmente no mercado local, sem certificação e fazendo pouco uso de crédito. Isso permite afirmar algo já esperado como resultado: empresas expostas a um ambiente de maior concorrência tendem a ajustar-se para os padrões competitivos do ambiente concorrencial, sob pena de serem excluídas do mercado.

Tabela 1- Estatísticas descritivas das variáveis independentes segundo as empresas que inovaram e as que não inovaram

\begin{tabular}{|c|ll|c|c|}
\hline \multirow{2}{*}{ VARIÁVEL } & \multicolumn{2}{|c|}{ CATEGORIAS } & \multicolumn{2}{|c|}{ PARTICIPAÇ̃̃O PERCENTUAL (\%) } \\
\cline { 3 - 4 } & & Inova (11) & Não Inova (25) \\
\hline \multirow{3}{*}{ Escolaridade } & $0-$ & Até ensino médio & 9,1 & 16,0 \\
& \multicolumn{2}{|c|}{ incompleto } & 18,2 & 28,0 \\
& $1-$ & Ensino médio & 72,7 & 56,0 \\
& $2-$ & Ensino superior & 27,3 & 64,0 \\
\hline Serraria & $1-$ & Sim & &
\end{tabular}

Revista de Administração e Inovação, São Paulo, v.12, n.1, p.248-270, jan./mar. 2015. 


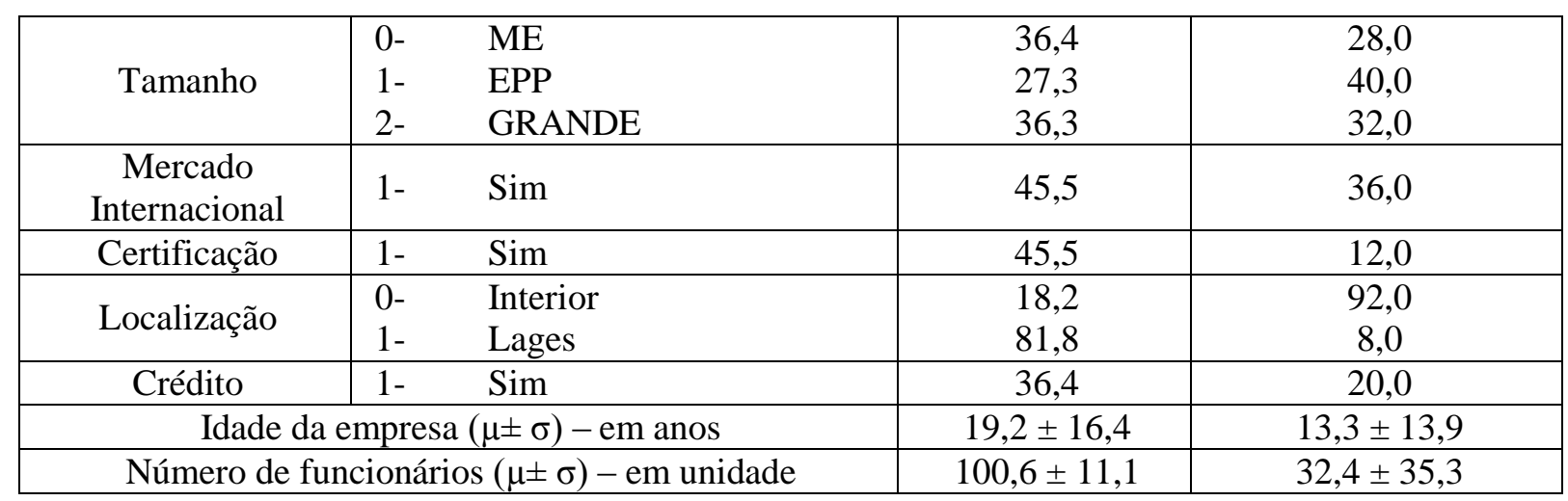

Fonte: Pesquisa de campo.

Visando identificar as variáveis que aumentam a probabilidade das empresas madeireiras adotarem inovação, apresenta-se o resultado das análises econométricas. Inicialmente, o modelo de regressão logística apresenta a equação que só considera o intercepto (constante) cujo valor $\beta_{0}=$ 0,8210, é estatisticamente diferente de zero pela estatística Wald $(5,1487)(\mathrm{P}<0,0233)$. Neste, as 25 empresas que não inovaram foram corretamente preditas (100\%) pelo modelo que continha apenas o intercepto. Entretanto, não houve a indicação de nenhuma das 11 empresas que realizaram alguma inovação. Sendo assim, com apenas o intercepto, o modelo previu corretamente 69,44\% das informações prestadas pelos gestores das empresas. Neste caso, o impacto do coeficiente sobre a chance de inovar é 0,44 .

Pelo método Forward Stepwise (razão de verossimilhança) as variáveis independentes são, sequencialmente, acrescidas ao modelo. Após sucessivas estimações, os resultados obtidos pela regressão logística evidenciam que apenas a variável "Localização" foi significativa para explicar a inovação tecnológica na indústria madeireira da região de Lages (Tabela 2). Houve significância estatística para os coeficientes de regressão logística das variáveis "Localização" e "Constante" $(\mathrm{P}<0,05)$, conforme indicado pelo teste de Wald.

Tabela 2- Coeficientes e significância das variáveis da regressão logística

\begin{tabular}{|c|c|c|c|c|c|}
\hline Variável & $\beta$ & S.E & Wald & df & Sig \\
\hline Localização & 3,4965 &, 9949 & 12,3502 & 1 &, 0004 \\
\hline Constante & $-2,3978$ &, 7385 & 10,5415 & 1 &, 0012 \\
\hline
\end{tabular}

A bondade de ajustamento é verificada pela estatística -2LL (teste de razão de verossimilhança) que reduziu de 44,32 no modelo que continha somente o intercepto, para 27,26 quando se inclui a variável Localização, resultando no valor de G (Goodness of Fit) de 35,999. Outra forma de avaliar a qualidade do modelo é através do $\mathrm{R}^{2}$ de Nagelkerke, cujo valor foi de 53,3\%. Neste modelo, das 25 
empresas que não inovaram, 22 foram corretamente preditas (88\%), enquanto que, das onze empresas que inovaram, o modelo previu corretamente nove delas $(81,82 \%)$, resultando em um percentual total de acerto de $86,11 \%$, significativamente superior ao modelo com tão somente o intercepto $(69,44 \%)$. Os resultados indicam que as empresas sediadas na cidade de Lages têm maior probabilidade de inovar, confirmando a hipótese inicial.

Lages constituiu-se, historicamente como cidade pólo da região da serra catarinense, tendo maiores indicadores sociais e econômicos, além de capacidade de articulação política, principalmente para captação de recursos. Por outro lado, os municípios do interior da região são menores e caracterizam-se por apresentar baixos indicadores de desenvolvimento econômico e social, os quais contribuem para limitação da possibilidade de desenvolvimento industrial. A concentração da indústria madeireira e metal mecânica na cidade de Lages contribui para o desenvolvimento de maior dinâmica empresarial/industrial, pela interação entre as firmas, desenvolvimento de infraestrutura, existência de organizações de apoio e oferta de mão de obra especializada.

Assim, as empresas sediadas em Lages diferenciam-se das demais, pois as condições de infraestrutura possibilitam a realização de ações coletivas para, por exemplo, criar consórcios de exportação ou compra de matérias-primas. Além disso, dispõem de instituições locais de qualificação de mão de obra e prestação de serviços especializados (bridge institutions), as quais incluem instituições capacitadas para P\&D focado para o setor (como é o caso das instituições de ensino superior e do SENAI). Este ambiente propicia estímulo à cooperação entre empresas sob alguma forma de governança (SUZIGAN, 2002). Deza (1995) também pondera que o contexto institucional (variáveis sociais, legais, institucionais, econômicas, entre outras) no qual se dá o processo de difusão, é importante para dinamizar o mesmo, o que pode estar ocorrendo localmente.

A análise multivariada mostrou que a dimensão um da PCA para a inovação tecnológica explicou 35,4\% da variabilidade dos dados, e as dimensões dois, três e quatro explicaram, respectivamente, $17,9 \%, 10,9 \%$ e 10,6\%, explicando $74,9 \%$ da variabilidade total dos dados. A porcentagem de resposta obtida pelas duas primeiras dimensões explicou, conjuntamente, 53,4\% da variabilidade total.

De maneira geral, a Figura 1 representa o valor médio dos componentes principais representados através dos centroides da variável dependente (Inovação) e mostra a nítida separação entre os dois grupos avaliados. O biplot da PCA indica a diferença das empresas que inovam das que não inovam, cujos centroides estão localizados em quadrantes diferentes e separados pelas dimensões um e dois.

Revista de Administração e Inovação, São Paulo, v.12, n.1, p.248-270, jan./mar. 2015. 
De acordo com a Figura 1, a inovação está fortemente associada à localização e ao aumento do tempo de vida da empresa, da utilização de crédito e da certificação, enquanto que a não inovação é verificada nas empresas que atuam no ramo das serrarias. Contudo, as demais variáveis analisadas (escolaridade, número de funcionários, mercado e tamanho), não apresentam tendência clara quanto aos seus efeitos sobre a inovação, tornando-se hipóteses não confirmadas, apesar da análise preliminar ter dado indicativos de uma relação positiva entre algumas destas variáveis e o comportamento inovativo.

\section{Figura 1- Variáveis explicativas e valor médio dos centroides dos grupos analisados através da}

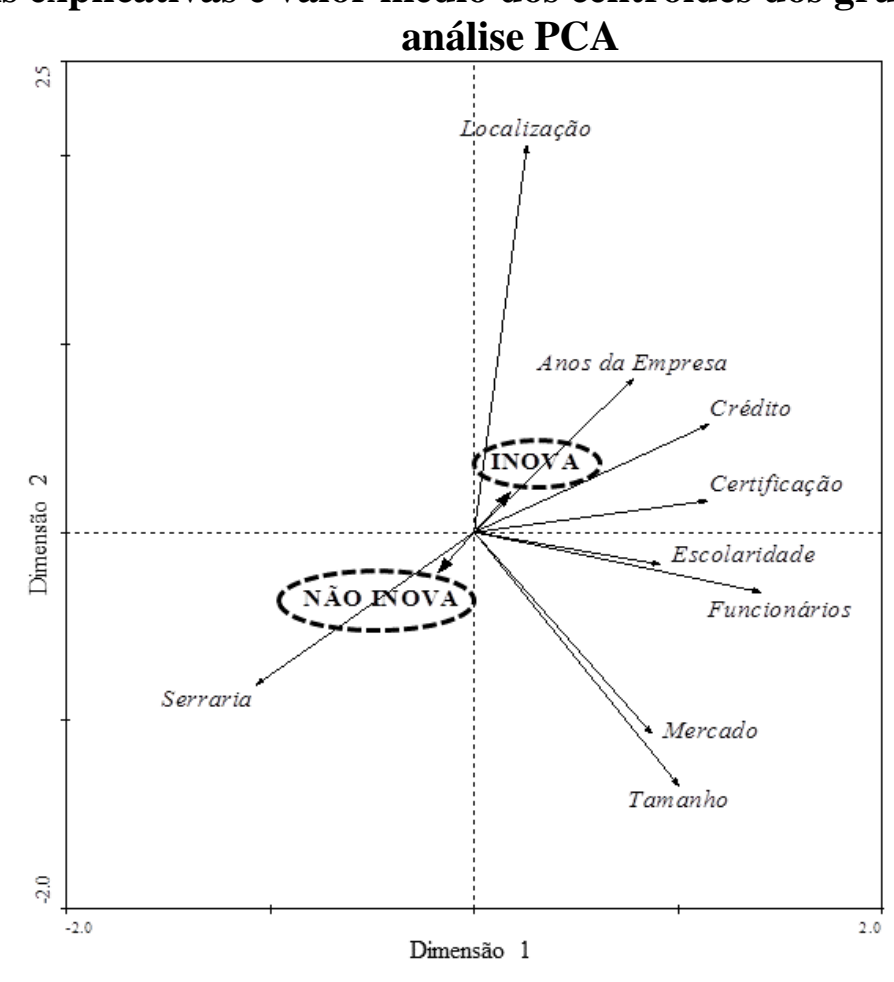

Fonte: Elaboração dos autores com base na pesquisa de campo.

Considerada a análise do modelo estatístico, é interessante detalhar um pouco mais os resultados obtidos na pesquisa de campo, indicando características do processo inovativo dentro das empresas. A partir da avaliação das mudanças adotadas entre as empresas que inovam, constata-se que são tipicamente pequenas e cotidianas, podendo ser classificadas como incrementais de acordo com a taxonomia dada por Freeman e Soete (1997). Para os autores, estas mudanças são derivadas, principalmente, do aprendizado interno e da capacitação acumulada. Melhorias feitas nos processos, em design, no layout e na qualidade são exemplos destas inovações (TIGRE, 2006).

O baixo valor agregado dos principais produtos comercializados ajuda a explicar o comportamento das firmas, pois neste ambiente concorrencial ainda não existem grandes pressões pela 
inovação e pela adoção de novas tecnologias. O modelo de regressão logística ratifica esta afirmativa, no momento que coloca as empresas do tipo "serraria" como fortemente, não inovadoras. Assim, o grau de maturidade das tecnologias utilizadas pode ser classificado como de tecnologias-base, de acordo com Waack (2000). Estas são amplamente dominadas pela concorrência e não dão acesso a vantagens competitivas. Este comportamento reflete constatações feitas em pesquisas anteriores. De acordo com Hoff e Simioni (2004) e com Hoff et al. (2006), o comportamento das empresas do setor de base florestal na região de Lages (SC) era caracterizado por baixos investimentos na diferenciação de produtos, onde a madeira serrada caracterizava-se como o principal produto produzido.

As serrarias regionais são empresas de pequeno porte, com administração familiar e reduzido número de funcionários, que produzem madeira serrada de primeira transformação, tais como tábuas brutas, ripas e barrotes ou com baixo grau de beneficiamento, como forros, rodapés e outras peças de madeira utilizadas na construção civil. Como identificado por Simioni, Brand e Hoff (2002), Hoff e Simioni (2004) e Hoff et al. (2006), é comum que estas empresas utilizem equipamentos instalados durante o primeiro ciclo da madeira na região (que se inicia na década de 1940 e finda na década de 1970), os quais tinham como foco o desdobramento da madeira nativa (araucárias, canelas, cedros) e que não estão totalmente adaptados para desdobro da madeira oriunda dos plantios florestais recentes (pinus e eucalipto, principalmente). Corroborando com isto, Manhiça et al. (2012) destacam que ainda é comum pequenas serrarias na região Sul do Brasil processarem a madeira usando equipamento lento e técnicas não adequadas. Isso justifica a busca de produtividade e redução de perdas e custos com a inovação tecnológica indicada pelos empresários. Essa informação reforça os resultados obtidos de que as serrarias e localização no interior estão associadas à NÃO INOVAÇÃO.

No entanto, as empresas que buscam certificação apresentam comportamento distinto do conjunto mais amplo. Para estas, investimentos em tecnologias que resultem em padronização da produção, com redução de variabilidade do produto, de perdas e com incremento de qualidade, é algo considerado como importante para o processo produtivo, pois confere-lhes maior competitividade. Mesmo assim, inovações ainda são consideradas pelos gestores entrevistados, como investimentos necessários ao processo e ao produto, desconsiderando-se a possibilidade de serem necessárias à melhoria da gestão.

Em relação ao alcance dos objetivos definidos na ocasião da implementação das inovações, constatou-se que a grande maioria das empresas (75\%) afirma que os objetivos traçados foram alcançados. No que se referem aos efeitos esperados pelas inovações, indicam em sequência de importância: melhoria da qualidade, redução de perdas, maior produtividade e redução de custos

Revista de Administração e Inovação, São Paulo, v.12, n.1, p.248-270, jan./mar. 2015. 
(Figura 2). Tais resultados podem impulsionar novos ciclos de inovação, uma vez que permitem ao gestor identificar objetivamente ganhos a partir do investimento.

Figura 2- Efeitos esperados da adoção de inovações tecnológicas pelas empresas

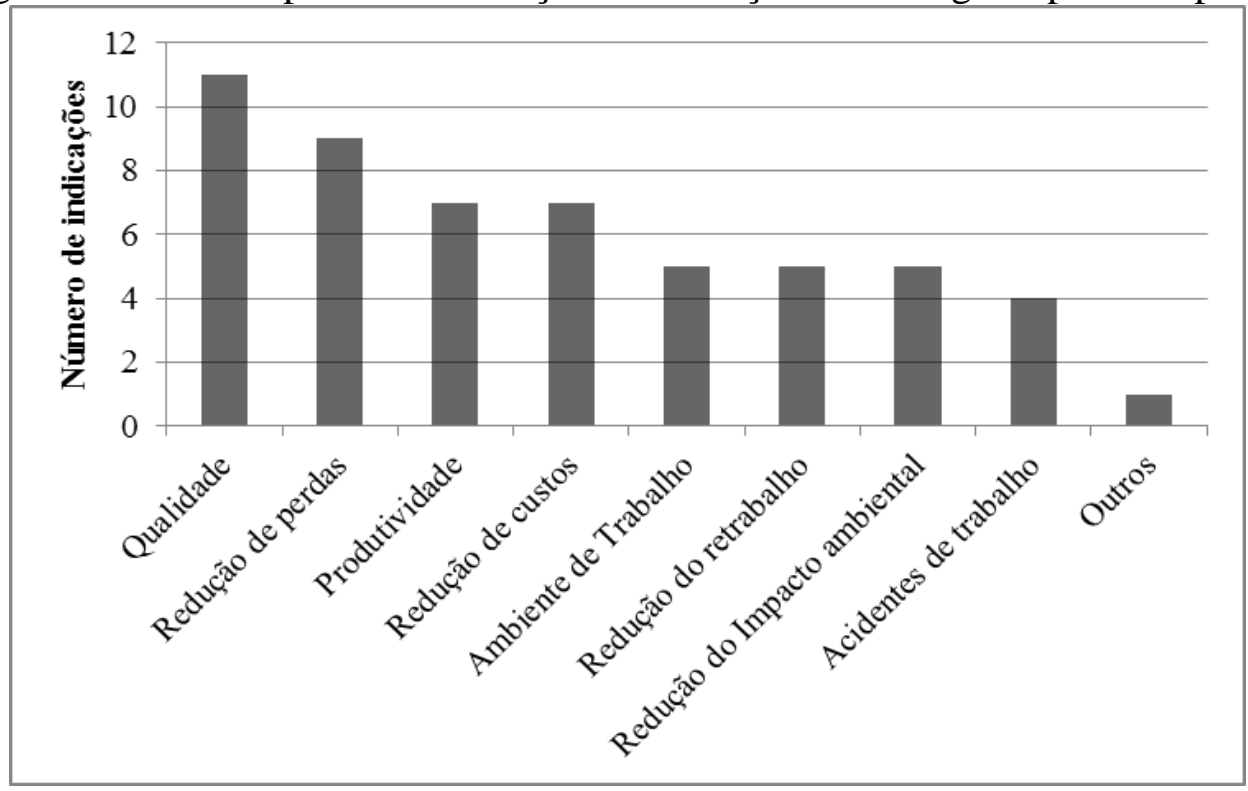

Fonte: Elaboração dos autores com base na pesquisa de campo.

Vale enfatizar que este tipo de efeito está muito mais ligado à superação de limites no processo produtivo existente do que à conquista de vantagens competitivas no mercado (sobretudo porque a diferenciação e a diversificação do portfólio de produtos não aparecem como efeitos esperados da inovação).

O fato do principal impulsionador da inovação ter sido a solicitação de clientes (Figura 3) corrobora a indicação de que o setor é reativo (responde ao que o mercado está pedindo), adotando tecnologias a partir das pressões de seus consumidores. Nesta perspectiva, o mercado produz estímulos para o desenvolvimento de processos inovativos que resultam em direções da tecnologia a ser adotada. Estes sinais são captados a partir das solicitações dos compradores sobre aspectos técnicos dos bens que desejam, ou pelo incremento no desejo por bens com maior tecnologia. A mudança técnica de processos e de produtos é a forma pela qual as firmas reagem às mudanças, visando atender aos seus clientes (DOSI, 1988; CÁRIO, 1995). 
Figura 3- Fatores que induziram a implantação da inovação tecnológica

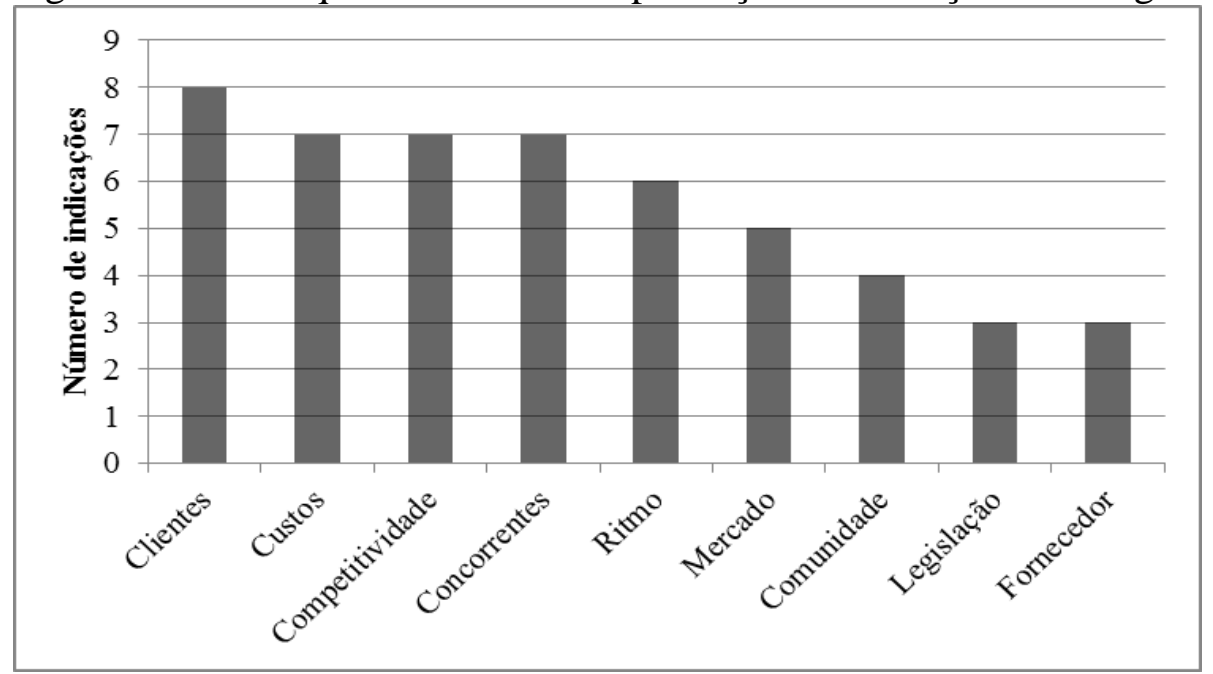

Fonte: Elaboração dos autores com base na pesquisa de campo.

Um dos fatores apresentados na Figura 3 é evidenciado por uma afirmativa identificada em seis respostas: "conforme a necessidade, se inova". Seguem, no mesmo nível de importância, fatores como redução de custos, maior competitividade e impulso gerado pelos concorrentes. Considerando-se que as empresas trabalham com produtos de baixa diferenciação, mesmo no grupo que inova, a disputa de mercado através de preços tende a ser um padrão competitivo.

Embora os dados sinalizem para a presença do mercado como fator impulsionador (demand pull), destaca-se que tais apontamentos estão relacionados a procedimentos de processo que conferem maior padronização e qualidade aos produtos, representando algo novo para a empresa, mas não para o mercado.

As análises também mostram coerência entre os fatores indutores da inovação e as pressões sofridas pela empresa no mercado. Isso é demonstrado por 14 empresas, as quais indicam que a necessidade de modernização, adoção de tecnologias e inovação está ligada à necessidade da empresa atuar no mercado competitivo. Investigando a literatura disponível sobre o tema, Sachuck et al. (2008), contataram que as inovações tecnológicas impactam a competitividade organizacional na medida em que reduzem os custos ou contribuem para a diferenciação dos negócios da empresa, otimizando a produtividade, melhorando a comunicação e a qualidade dos produtos, bem como as técnicas de gestão empregadas.

Por serem empresas de menor porte, era esperado que o alto custo das novas tecnologias fosse um dos limitantes de sua adoção (com 10 indicações). Vale enfatizar que a preocupação com custos e competitividade é forte entre os que adotam inovações. Isso pode ser considerado positivo, porque 
denota uma preocupação com a concorrência e com a necessidade de ajustar a organização para a disputa de mercado.

O segundo principal fator limitante refere-se à dificuldade de desenvolvimento de novas tecnologias para o setor produtivo. Destacam-se também outros dois fatores limitantes intimamente relacionados: dificuldade no uso das novas tecnologias e falta de mão de obra qualificada para operar as tecnologias disponíveis.

As mudanças tecnológicas introduzidas concentraram-se, principalmente, no layout, nos sistemas de controle e na comercialização. Segue a estes, em ordem decrescente, a tecnologia inserida pela incorporação de máquinas e pela aquisição de insumos de matéria prima. De acordo com OCDE (2004) e Tigre (2006), as inovações empregadas pelas firmas são classificadas como "de processo".

Quando questionados sobre a maior necessidade de tecnologia, os pesquisados indicaram a introdução de maquinários e incrementos de automação das fábricas. Este tipo de tecnologia tende a produzir os efeitos esperados da adoção tecnológica, conforme mencionado anteriormente (Figura 2).

Por fim, não se pode dizer que a observação feita sobre a realidade encontrada permita analisar que o mesmo esteja ou não em uma condição path dependent. A análise histórica do setor, em âmbito regional indica que escolhas tecnológicas feitas em determinados momentos podem estar limitando o desenvolvimento e a adoção de inovações. Por outro lado, a existência de um processo de desenvolvimento, de longo prazo, do setor de base florestal, na região, permite observar o desenvolvimento de conhecimento tácito e expresso sobre o setor e seus processos, assim como de instituições capazes de formar mão de obra (do chão de fábrica aos cargos gerenciais), de marco institucional específico, de políticas públicas focadas, de organizações capazes de desenvolver P\&D para o setor. Estas percepções podem indicar a existência de path dependence no setor. No entanto, afirmativas mais contundentes, neste sentido, dependeriam de aprofundamento de conhecimento sobre o tema, inclusive acerca do comportamento do mesmo em âmbito mundial, quando considerado este aspecto, tal como feito por Hoff (2008) com a indústria de celulose e papel.

Parece ser mais coerente, pelas informações obtidas, dizer que este comportamento é característico do setor: que é reativo ao comportamento da concorrência e dos consumidores (que geralmente são outras firmas) quando estes exigem mudanças, mas que ainda não usa fortemente a adoção da inovação como fator criador de vantagem competitiva sobre os concorrentes. Este padrão de comportamento do setor também foi apontado por Pelaez et al. (2009), onde verificaram que as decisões de indústrias de máquinas para madeira "são baseadas exclusivamente num padrão de comportamento reativo que não inclui atividades de planejamento nem a elaboração de cenários 
prováveis ou desejáveis". Estudos internacionais indicam comportamento diverso de empresas em ambientes mais competitivos, conforme pode ser observado em Wagner e Hansen (2005).

\section{CONCLUSÕES}

De modo geral, os dados indicaram que as empresas, independente do tamanho, enfrentam dificuldades em inovar, sobretudo em produtos e a tendência é que esse desenvolvimento ocorre quando há uma demanda específica do mercado em que a empresa atua. Mesmo assim, o limite da inovação encontra-se nos ganhos incrementais em tecnologias de base, relacionadas, principalmente ao processo produtivo. Com isso, não há o uso das inovações como elementos fundantes de estratégias competitivas. Quando usada em benefício do estabelecimento de uma estratégia competitiva, a inovação tecnológica tende a contribuir para controle e redução de custos, muito mais do que diferenciação ou diversificação do produto.

Cerca de um terço das empresas estudadas realizaram algum tipo de inovação tecnológica no período de estudo, inseridas principalmente no processo produtivo. A localização das empresas em um ambiente externo mais competitivo é a principal variável que contribui para o aumento da probabilidade de inovar. A inovação também está fortemente associada ao aumento do tempo de vida da empresa, à utilização de crédito e à busca da certificação pelas empresas. Por outro lado, a não inovação está fortemente associada às serrarias.

Melhoramentos e modificações cotidianas caracterizam o grau de inovação e a extensão das mudanças em relação ao que havia antes como incrementais. Porém, o grau de maturidade das tecnologias é classificado como tecnologias-base, uma vez que são amplamente difundidas entre as empresas do setor. As inovações tecnológicas são, grandemente, incorporadas pelas empresas a partir das solicitações dos clientes, estando relacionadas à melhoria da qualidade dos produtos e à redução dos custos de produção.

Por fim, as empresas seguem o padrão de comportamento da indústria madeireira nacional. Uma comparação entre as observações feitas em âmbito nacional com a realidade internacional do setor, que parece ser distinta, é um indicativo de pesquisa futura.

Revista de Administração e Inovação, São Paulo, v.12, n.1, p.248-270, jan./mar. 2015. 


\section{REFERÊNCIAS}

BARNEY, J. B. Organizational Culture: can it be a source of sustained competitive advantage? Academy of Management Review. v. 11, n. 3, p. 656 - 665, 1986.

CÁRIO, S. A. F. Contribuição do paradigma microdinâmico neo-schumpeteriano à teoria econômica contemporânea. In: Universidade Federal de Santa Catarina (org.). Textos em economia. Florianópolis, UFSC, 1995.

CASSIOLATO, J. Innovacion y cambio tecnológico. In: MARTINEZ, E. (ed.). Ciência, tecnologia y desarrollo: interrelaciones teóricas y metodológicas. Chile: Nova Sociedad, 1994.

DOSI, G. Sources, procedures and microeconomic effects of innovation. Journal of Economic Literature, v. 26, n. 3, p. 1120-1171, 1988.

FIGUEIREDO, P.N. Aprendizagem tecnológica e inovação industrial em economias emergentes: uma breve contribuição para o desenho e implementação de estudos empíricos e estratégias no Brasil. Revista Brasileira de Inovação. Rio de Janeiro, v.3, n.2, p.323-362, jul./dez. 2004.

FREEMAN, C.; SOETE, L. The economics of industrial innovation. Cambridge (MA): MIT Press, 1997.

HEDBERG, B. How Organizations Learn and Unlearn. In: NYSTROM, P.C.; STARBUCK, W.H (eds.). Handbook of Organizational Design. New York: Oxford University Press, p. 3 - 27, 1981.

HOFF, D.N. A construção do desenvolvimento sustentável através das relações entre as organizações e seus stakeholders: a proposição de uma estrutura analítica. 2008 . $426 \mathrm{f}$. Tese (Doutorado em Agronegócios) - Programa de Pós-Graduação em Agronegócios, Centro de Estudos e Pesquisas em Agronegócios, Universidade Federal do Rio Grande do Sul, Porto Alegre, 2008.

HOFF, D. N.; SIMIONI, F. J. O setor de base florestal na serra catarinense. Lages: Editora Uniplac, 2004. $268 \mathrm{p}$.

HOFF, D. N.; SIMIONI, F. J.; BRAND, M. A. Análise da competitividade da indústria de base florestal da região de Lages, SC. Ensaios FEE, v. 27, n. 1, p. 109-134, maio 2006.

IBGE - INSTITUTO BRASILEIRO DE GEOGRAFIA E ESTATÍSTICA. Pesquisa de inovação tecnológica: 2008. Rio de Janeiro: IBGE, 2010. 164 p.

MANHIÇA, A. A.; ROCHA, M. P. DA; TIMOFEICZYK JÚNIOR, R. Rendimento no desdobro de Pinus $s p$. utilizando modelos de corte numa serraria de pequeno porte. FLORESTA, Curitiba, PR, v. 42, n. 2, p. 409 - 420, abr./jun. 2012.

NELSON, R.; WINTER, S. Introduction. In: A evolutionary theory of economic change. EUA: Harvard Press, 1982.

OCDE. Manual de Oslo: proposta de diretrizes para coleta e interpretação de dados sobre inovação tecnológica. OCDE/FINEP, 2004. 
PELAEZ, V.; AQUINO, D.; HOFMANN, R. M.; MELO, M. F. Análise da capacidade dinâmica na indústria de máquinas para madeira: estudos de caso. Revista Brasileira de Inovação, Rio de Janeiro (RJ), v. 8, n. 2, p.341-370, julho/dezembro, 2009.

POLANYI, M. The Tacit Dimension. N.Y: Publisher Garden City, 1967.

POSSAS, M. S. Concorrência e competitividade: notas sobre estratégia e dinâmica seletiva na economia capitalista. Campinas: UNICAMP, 1993. Cap. II, p. 60-102. (Tese de Doutorado).

POSSAS, M. Concorrência schumpeteriana. In: KUPFER, D.; HASENCLEVER, L. Economia industrial: fundamentos teóricos e práticos no Brasil. Rio de Janeiro: Campus, 2002.

RIBAS, J. R.; VIEIRA, P. R. da C. Análise multivariada com uso do SPSS. Rio de Janeiro: Editora Ciência Moderna, 2011.

SACHUCK, M. I.; TAKAHASHI, L. Y.; AUGUSTO, C. A. Impactos da inovação tecnológica na competitividade e nas relações de trabalho. Caderno de Administração, v. 16, n. 2, p. 57-66, jul/dez. 2008.

SILVA, C.R.L.da. Inovação tecnológica e distribuição de renda: impacto distributivo dos ganhos de produtividade da agricultura brasileira. São Paulo: IEA, 1995.

SIMIONI, F. J.; BINOTTO, E.; HOFF, D. N. Tecnologia e inovação na indústria madeireira da região de Lages. Lages: FAPESC/UNIPLAC, 2010. 73 p. (Relatório de Pesquisa).

SIMIONI, F. J.; BINOTTO, E.; NERI, A. C.; SIQUEIRA, E. S. Reflexões a respeito do conceito de cadeia produtiva no agronegócio florestal. In: BINOTTO, E. (Org.) Tecnologia e processos agroindustriais. Passo Fundo: Editora da Universidade de Passo Fundo, 2007, p. 97-116.

SIMIONI, F. J.; BRAND, M. A.; HOFF, D. N. Caracterização do cluster da madeira da região de Lages/SC. Lages, 2002. (Relatório de Pesquisa).

SIMIONI, F. J.; BINOTTO, E.; HOFF, D. N. A inovação tecnológica na perspectiva dos gestores da indústria madeireira da região de Lages, SC. Revista Árvore, Viçosa-MG, v. 36, n. 5, p.981-987, 2012.

SIMON, H. A. Administrative Behavior: a study of decision making process in administrative organization. New York: Macmillan, 1957.

STARBUCK, W. H. Organizations as Action Generators. American Sociological Review. v. 48, n. 1, p. $91-102,1983$.

SWIERING, J.; WIERDSMA, A. La Organización que Aprende. Wilmington, USA: AddisonWesley, 1995.

TER BRAAK, C. J. F.; SMILAEUR, P. C. Reference manual and user's guide to canoco for windows: Software for Canonical Community Ordination (version 4), Ithaca, New York, USA, Microcomputer Power. 1998.

TIGRE, P. Gestão da inovação: a economia da tecnologia no Brasil. Rio de Janeiro: Elsevier, 2006.

Revista de Administração e Inovação, São Paulo, v.12, n.1, p.248-270, jan./mar. 2015. 
WAACK, R. S. Gerenciamento de tecnologia e inovação em sistemas agroindustriais. In: ZYLBERSTAJN, D. NEVES, M. F. Economia e gestão dos negócios agroalimentares: indústria de alimentos, indústria de insumos, produção agropecuária, distribuição. São Paulo: Pioneira, 2000. 428p.

WAGNER, E.; HANSEN, E. N. Innovation in large versus small companies: insights from the US wood products industry. Management Decision, v. 43, n. 6, p. 837-850, 2005.

WALSH, J. P.; UNGSON, G. R. Organizational Memory. Academy of Management Review. v. 16, n. 1, p. 57 - 91, 1991.

WEICK, K. E. The Nontraditional Quality of Organizational Learning. Organization Science. v. 2, n. 1, p. 116 - 124, 1991.

\begin{abstract}
Objective to analyze the variables that affect the adoption of technological innovations of wood industry in the region of Lages/SC, its characteristics and the default behavior of firms when the innovation. The research included 36 companies and data were collected with a questionnaire and submitted to logistic regression analysis (logit) and the Principal Component Analysis (PCA). The results indicate that innovation is linked to location in a more competitive external environment, the increase of the time, the use of credit and the pursuit of certification by companies, while the sawmills are associated to innovation. The technologies adopted are diffusion in the industry.
\end{abstract}

Key-words: Technology; Companies; wood; path dependence.

Data do recebimento do artigo: 03/05/2014

Data do aceite de publicação: 15/01/2015

Revista de Administração e Inovação, São Paulo, v.12, n.1, p.248-270, jan./mar. 2015. 